\title{
Genain Quaduplets: Variations on the Schizophrenic Syndrome
}

\author{
Nancy L. Segal \\ Department of Psychology, California State University, USA
}

Genain is the pseudonym for a rare monozygotic female quadruplet set, famous for their schizophrenia concordance. Derived from the Greek language, Genain means "dreadful birth" or "dire gene" (Rosenthal, 1963). Most investigators now agree that schizophrenia represents a complex interaction among genetic, pre-natal, psychosocial and random events, although they disagree over the relative contributions of each one (Moran, 2000).

The Genain quadruplets were born in an undisclosed midwestern town on April 14, 1930. They were intensively studied for a three and a half-year period (1955-1958) by an interdisciplinary team at the National Institute of Health, lead by the late Dr. David Rosenthal. In order to disguise their identity, the quads were cleverly called Nora, Iris, Myra and Hester, names whose first letters correspond to NIMH and whose sequence follows the quads' order of birth. This decision is known by people familiar with their story. A less well-known fact is that it continued a practice begun by their parents - the quads' real names follow letters in the name of the hospital in which they were born (A.F. Mirsky, personal communication, 2 January, 2001)

Research efforts culminated in a seminal book, The Genain Quadruplets, published in 1963 and edited by Rosenthal (1963). The dedication is heartfelt, acknowledging a psychiatrist, a historian, an arts patron, a war hero and a father whose untimely deaths left plans and hopes unrealized - "And the mentally afflicted everywhere, nameless, the lost living, their talents bud-nipped, their dreams imprisoned, their songs unsung, who, in a more grievous sense, died too young" (p. v).
Beyond its subject matter, the volume is memorable for Rosenthal's skilled assembly of 37 separate chapters reflecting his and his colleagues' professional specializations. Follow-up visits resulted in new research findings published in 1984, 1998, 1992, 1997 and 2000 (Buchsbaum et al., 1984; Delisi et al., 1984; Jönsson et al, 1997; Mirsky \& Quinn, 1988; Mirsky et al, 1984, 1992, 2000). The final paper especially caught my attention as it underlined the continued research potential of the sisters, now in their seventies. It also recalled another time and place. I assisted David Rosenthal at the NIMH during the summer of 1974 by organizing information in letters he received from Nora, Iris, Myra and Hester. (Yes, I know their real names!) This was an exciting task for a first-year graduate student for several reasons. Twin research was flourishing and refining genetic explanations of schizophrenia. The Genain quadruplets' scientific

\section{"His new research findings on the Genain quadruplets cognitive capacities bear importantly on current conceptions of cognitive performance in schizophrenic patients."}

story was, by then, a classic, studied by thousands of people around the world. The opportunity to read and code the quads' written words lent richness and vitality to the collected works I had just studied. Now, thinking back over that time, I reached again for my well-worn copy of The Genain Quadruplets. I also contacted Dr. Allan F. Mirsky,
Chief of the Section on Clinical and Experimental Neuropsychology, Laboratory of Brain and Cognition, NIMH, and first author of the 2000 paper. Mirsky was an associate of the study since its inception, specializing in neuropsychological assessment. His new research findings on the Genain quadruplets cognitive capacities bear importantly on current conceptions of cognitive performance in schizophrenic patients. These data are presented below, followed by Dr. Mirsky's unique professional and personal reflections on the project. They are the "stuff" that research is made of...

\section{The Genain Quaduplets: \\ Past and Present}

The birth of MZ quadruplets is a rare event, estimated to occur in one in ten million births (Allen et al., 1960). The monozygosity of the Genain quadruplets was established by concordance across eight blood group systems and other heritable traits (Rosenthal et al., 1963). The sisters had, for example, inherited the same hair color (blonde) and skeletal anomaly (extra epiphyhsis at the base of the first metacarpal bone). The probability of dizygosity based on these features is less than one per cent. Inspection of the chorionic sac suggested it was shared by at least three of the quads, perhaps all four. Some dermatoglyphic differences were observed, most notably a total ridge count discrepancy of 41 between Hester and Iris, but other dermatoglyphic features did not show this degree of difference. Using qualitative and quantitative traits and organizing the quads into all possible two-egg combinations (e.g., two twin pairs; one triplet set with a co-quad) yielded probabilities of dizygosity ranging from 0.072 to 0.0008 . In 1997 find- 
ings of a cytogenetic abnormality in Swedish MZ male triplets was reported (Jönsson, Härnyrd et al, 1997). This same finding was not replicated in the Genain quadruplets (Jönsson, Mirsky et al., 1997).

Schizophrenia affects approximately $1 \%$ of individuals (Gottesman, 1991). The estimated probability of finding identical quadruplet sisters all developing the disorder was one in one and a half billion births (Rosenthal, 1963). Despite the long odds of this event, the fact that Nora, Iris, Myra and Hester differed in age of onset, symptom expression and symptom severity may be more compelling. Their discrepancies are most likely associated with differences in brain trauma, parental treatment and other sources, although assigning relative causal roles to each one is virtually impossible. Behavioral and physical variations in these $M Z$ quadruplets continue to interest and challenge the researchers who follow them faithfully.

Neurological tests were administered to three of the four Genain sisters in 1996 when they were age 66 years (Mirsky et al., 2000). Iris was suffering from dementia with agitation and schizophrenia at that time so was unable to participate. Tests included the digit span, arithmetic and digit symbol subtests from the Wechsler Adult Intelligence Scale-Revised (WAIS-R), the Talland Letter Cancellation Test, the Trail Making Test, the Wisconsin Card Sorting Test and the Visual X, Visual AX and Auditory Tones Tasks from the Continuous Performance Test (CPT). A version of the CPT had also been completed by the quads in 1957 and 1981 when they were 27 and 51 years of age, respectively. Data from these three time points, therefore, enabled tracking and comparison of the sisters' levels of sustained attention; deficits in this capacity have been linked to schizophrenia by some, but not all, investigators. The CPT data are emphasized in the 2000 follow-up report and in this review. $Z$ scores for each test were computed for each sister and compared with means generated by 27 healthy adults in their age range. Most of the Genains' scores fell over one standard deviation below the control means. However, the much higher educational level of the compar- ison group precluded clear interpretation of these differences. Subsequent comparisons with a less well-educated group also revealed reduced performance by the quads. Again, the relative contributions of the sisters' illnesses, educational histories and intellectual capacities to their test performance proved difficult to disentangle.

Myra, always the healthiest of the four, scored highest on measures assessing sustained and shifting attention.

\section{“The Genain quadruplets' life histories are vital reminders that human \\ behavioral variations are complex and multiply determined."}

Her performance was lower on several reaction time tests, possibly linked to her extended treatment with neuroleptic medication. Nora's scores generally resembled those of Hester (the most debilitated sister) more than those of Myra. This pattern of performance agreed with indications of greater early brain damage in these two sisters, relative to Myra and Iris. This "pairing" among the quads differs from that recognized by their parents who placed Nora with Myra, and Hester with Iris. The latter organization was based on Nora and Myra's earlier physical development, relative to Iris and Hester. Iris was developmentally more like Nora and Myra than Hester and displayed the best verbal skill. Nevertheless, their mother identified Nora and Myra as a favored, "smarter" pair, keeping them on her left side during infant feedings (Rosenthal, 1963, p. 49). The extent to which Iris's preemption by Myra may have exacerbated her schizophrenic predisposition cannot be fully known.

Mirsky and his colleagues (2000) noted that sustained attention is a sensitive measure of both schizophrenia deficit and risk. The four sisters performed consistently across the relevant CPT tasks in 1957 at age 27 years: Myra scored highest (within normal limits on one test), followed by Nora, Iris and Hester; Iris did quite poorly and Hester was unable to respond. The
CPT was repeated in 1981 when the quads were 51 years of age. Nora and Iris's scores increased, while Myra's scores variously declined across tasks. It was suspected that neuroleptic medication contributed to Nora and Iris's improved performance. (They were far more debilitated than Myra, so medication was beneficial.) In contrast, excessive medication may have caused Myra's performance to fall, given that her scores showed slight increases or decreases during medication-free intervals. (Myra was the healthiest of the four sisters, so it is thought that prolonged medication impaired her performance.) Finally, CPT scores were available for Nora, Myra and Hester in 1996 at age 66 years. Nora and Myra scored within normal limits on one of the tasks, while Hester's score was unchanged since 1981. Hester's quick reaction time at both test dates was thought to reflect her impulsive response style.

What insights do the Genain quaduplets now lend to our understanding of schizophrenia and its prognosis? First, the sisters' stability and occasional improvement in test scores show that they are "more than holding their own" (p. 705). This result is attributed, in part, to the availability of more effective medications and to the supportive climate provided to them by their community. Most importantly, this outcome challenges the notion that cognitive decline is an inevitable feature of the schizophrenic process. Secondly, the Genain quadruplets' life histories are vital reminders that human behavioral variations are complex and multiply determined. Fortunately, natural experiments of birth, especially MZ and DZ twins, triplets, quadruplets and more, can assist scientists in reducing this complexity.

Dr. Allan F. Mirsky (personal communication, 21 December 2000 and 2 January 2001) brought unique personal perspectives to his work with the Genain quadruplets for which I am grateful. When word of the Genain quadruplets was received, Rosenthal urged all members of the intramural program at $\mathrm{NIMH}$ to participate in the project, so Mirsky was involved since the beginning. He recalls testing "four slim young women" who differed in their degree of apprehension toward him. He has, 
however, extended his early work on the quads' sustained attention via the follow-up studies described above. $\mathrm{He}$ also recalled comments and conversations with the sisters over the years, some humorous, that offer insights into their personalities as well as their illnesses. One sister volunteered an especially memorable denunciation of a stimulus presented prior to a PET scan; interested individuals can contact this author for the "raw" data.

We can look forward to further findings from the Genain quadruplets. Plans include brain and blood studies that may reveal new clues to the onset and course of their disorder. I most enjoyed Mirsky's response to my initial inquiry:

"How nice to hear from another Genain researcher! I saw the sisters for the first time in 1958 or 1959 , or thereabouts, when we were all in our 20s. Now, mirabile dictu, we are all in our 70s. I visited them several weeks ago (in November, 2000) in Michigan and had a marvelous time. We brought along a group of neuropsychology trainees from the
$\mathrm{U}$ of Michigan, and as usual the sisters were gracious and welcoming. They sang for us "My sweet little Alice blue gown", something they had been doing for last $60+$ years. It nearly brought me to tears. I do think that they are a kind of national research treasure, and because of their kindness and cooperativeness, we continue to learn more about this most enigmatic of all human disorders."

P.S. I think of Dave Rosenthal often, and his wonderful research legacy.

\section{Research Reviews}

\section{Zygosity Diagnosis in Chimpanzee Twins}

The incidence of twinning among nonhuman primates offers insights into the nature and origins of human twinning (Segal et al., 2000). It is generally presumed that twinning among close human ancestors is dizygotic because monozygotic twinning reduces genetic variability among offspring. Furthermore, DZ twinning is partly genetically mediated. Given the hazards associated with multiple births, twinning among most nonhuman primates is rare and pairs born in the wild generally do not survive. (Exceptions include marmosets and tamarins, small primates from Central and South America, among whom twinning occurs commonly). Currently, the relative frequencies of $\mathrm{MZ}$ and DZ pairs among nonhuman primates remain uncertain.

The birth of chimpanzee twins, Teeone and Teetoo, in June, 1996, at Kansas's Sunset Zoo attracted considerable research interest (Zhang et al., 2000). DNA extracted from blood samples provided by the father (Big Mac), mother (Rachel) and twins was examined via study of microsatellites. (Microsatellites are tandem repeats of short nucleotide motifs. They are wellsuited to pedigree analysis because of high allelic diversity at each locus.) Primers developed for humans were used by California researchers to screen 34 microsatellite loci of the twins, of which 25 loci proved useful. The prob- ability of dizygosity was calculated as $3.7 \times 10^{-11}$, indicative of $\mathrm{MZ}$ twinning.

Debate surrounding the bases of $M Z$ twinning and its relationship to DZ twinning may be reinvigorated in light of the twins' mother's family history. Rachel was a twin herself and the twins' grandmother delivered six sets of twins, two pairs known to have been DZ. The possibility of multiple influences on $\mathrm{MZ}$ twinning (e.g., genetic factors, random processes) within different families remains unsolved.

\section{Communication Disorders in Schizophrenia}

Traits signaling genetic vulnerability to schizophrenia can facilitate early diagnosis and treatment of this disorder. A recent twin study of communication disorders may be breaking new ground (Docherty and Gottesman, 2000). The sample used schizophrenic MZ and DZ twins, their non-schizophrenic cotwins and twins from non-intact pairs who had participated in the wellknown Maudsley study. Twins' speech samples were analyzed by the Communications Disturbances Index (CDI), which yields scores for six types of unclear speech references. Three speech characteristics, thought to be associated schizophrenia were targeted: missing information references, ambiguous word meanings and structural clarities. An Object Sorting Task (OST), in which participants group objects together and justify objects' associations, was also administered. Key findings were that (1) missing information references occurred more frequently in the speech samples of $\mathrm{MZ}$ than DZ non-schizophrenic cotwins, and (2) missing references showed higher associations with OST performance than with the other two language problems. The researchers suggested that inheriting more severe forms of the specific speech deficit may affect vulnerability to schizophrenia. Individuals who are "preschizophrenic" may make frequent language mistakes, perhaps as many as do schizophrenics. This possibility could be tested with high-risk samples.

\section{Proposal: Breast Cancer and Co-Twin Control}

An unpublished study has suggested links between bra wearing and breast cancer (Poole, 2000). One hundred non-twin women suffering breast pain did not wear bras for a three-month period. Pre-menopausal participants experienced a $7 \%$ reduction in days of breast pain, while post-menopausal women did not. The investigator speculated that the garment may suppress the lymphatic system — vessels removing toxic waste from the body. This could conceivably lead to an accumulation of toxins in breast tissue. Of course, further efforts are needed to confirm these very preliminary findings and conjectures. Co-twin control studies in which one twin wore a bra and the 